\title{
FUNCTIONS HARMONIC IN A STRIP
}

D. V. WIDDER

1. Introduction. The form of the Poisson kernel appropriate for an infinite strip is undoubtedly familiar to many analysts. However, there seems to be no convenient reference to it; compare [1] and [2]. Hence a brief discussion of it here may not be without interest. If we set

$$
P(x, y)=\frac{\sin x}{\cosh y-\cos x},
$$

then a formal solution of the Dirichlet problem for the strip $0 \leqq x \leqq \pi$, $-\infty<y<\infty$, is

$$
\begin{aligned}
u(x, y)= & \frac{1}{2 \pi} \int_{-\infty}^{\infty} P(x, t-y) u(0, t) d t \\
& +\frac{1}{2 \pi} \int_{-\infty}^{\infty} P(\pi-x, t-y) u(\pi, t) d t .
\end{aligned}
$$

We supply a set of conditions sufficient for the validity and uniqueness of this representation. We also discuss positive harmonic functions, obtaining the analogue of the classical theorem of Herglotz. See $[3 ; 5]$.

2. Properties of the kernel. Let us use the letters $H, L, C$ to denote the classes of functions which are harmonic, Lebesgue integrable, and continuous, respectively.

Lemma 1. If $P(x, y)$ is the function (1) and $\delta$ is a positive number, then
A.
$P(x, y) \in H$
$-\infty<y<\infty, 0<x<2 \pi$
B.$$
P(x, y)>0
$$$$
0<x<\pi
$$
C.

$$
|P(x, y)| \leqq \frac{|\sin x| e^{-|y|+\delta}}{\cosh \delta-1}
$$$$
|y| \geqq \delta, 0 \leqq x \leqq 2 \pi ;
$$
D. $\quad \int_{-\infty}^{\infty} P(x, y) d y=2(\pi-x)$

$$
0<x<\pi
$$

The proofs of these facts follow from the following relations:

Received by the editors March 30, 1960. 

A. $\quad P(x, y)=2 \operatorname{Im}\left[e^{-i(x+i y)}-1\right]^{-1}$;
B. $\cos x<\cosh y$;
C. $\cos x<1, e^{-|y|}(\cosh y-1)$ is increasing for $\delta \leqq y<\infty$ and is even;
D. $\frac{d}{d y} \operatorname{ctn}^{-1}\left(\frac{\cos x-e^{y}}{\sin x}\right)=\frac{P(x, y)}{2}$.

By use of this lemma we now prove our first theorem.

Theorem 1. If $\phi(t) e^{-|t|} \in L$ on $(-\infty, \infty), \phi(t) \in C$ at $t=y_{0}$, and

$$
F(x, y)=\frac{1}{2 \pi} \int_{-\infty}^{\infty} P(x, t-y) \phi(t) d t
$$

then

$$
\begin{array}{lrrl}
\text { A. } & F(x, y) & \in H & -\infty<y<\infty, 0<x<2 \pi \\
\text { B. } & & F(\pi, y)=0 & -\infty<y<\infty \\
\text { C. } & \lim _{x \rightarrow 0+, y \rightarrow y_{0}} F(x, y)=\phi\left(y_{0}\right) . &
\end{array}
$$

In conclusion $\mathrm{C}$, the approach of $(x, y)$ to $\left(0+, y_{0}\right)$ is two-dimensional. The integral (3) converges uniformly in $0<x<2 \pi,|y| \leqq R$ for every $R>0$. For, in this region we have by conclusion $\mathrm{C}$ of Lemma 1 that

$$
\begin{aligned}
\int_{|t| \geqq R+\delta} P(x, t-y) \phi(t) d t & \ll e^{\delta} \int_{|t| \geqq R+\delta} \frac{|\phi(t)| e^{-|t-y|} d t}{\cosh \delta-1} \\
& \ll \frac{e^{R+\delta}}{\cosh \delta-1} \int_{|t| \geqq R+\delta}|\phi(t)| e^{-|t|} d t .
\end{aligned}
$$

This proves conclusions $\mathrm{A}$ and $\mathrm{B}$.

Given $\epsilon>0$, we choose $\delta$ so that $\left|\phi\left(y+y_{0}\right)-\phi\left(y_{0}\right)\right|<\epsilon / 2$ when $|y|$ $\leqq 2 \delta$. Then for $\left|y-y_{0}\right| \leqq \delta$ and $|t| \leqq \delta$

$$
\left|\phi(t+y)-\phi\left(y_{0}\right)\right|<\epsilon / 2 \text {. }
$$

For $0<x<\pi$ and $\left|y-y_{0}\right| \leqq \delta$ we have by B, C, D of Lemma 1 that

$$
\begin{aligned}
\mid F(x, y)- & \frac{(\pi-x)}{\pi} \phi\left(y_{0}\right)\left|\leqq \frac{1}{2 \pi} \int_{-\infty}^{\infty} P(x, t)\right| \phi(y+t)-\phi\left(y_{0}\right) \mid d t \\
& \leqq \frac{\epsilon}{4 \pi} \int_{-\delta}^{\delta} P(x, t) d t+\frac{\sin x}{2 \pi} \int_{|t| \geq \delta} \frac{e^{-|t|+\delta}\left|\phi(y+t)-\phi\left(y_{0}\right)\right| d t}{\cosh \delta-1} \\
& \leqq \frac{\epsilon}{2}+K \sin x,
\end{aligned}
$$


where

$$
K=\frac{\left|\phi\left(y_{0}\right)\right| e^{\delta}}{\pi(\cosh \delta-1)}+\frac{e^{\left|y_{0}\right|+2 \delta}}{2 \pi(\cosh \delta-1)} \int_{-\infty}^{\infty} e^{-|t|}|\phi(t)| d t .
$$

We have used the fact that

$$
\begin{gathered}
\int_{-\infty}^{\infty} e^{-|t|} d t=2, \\
\int_{|t-y| \geq \delta} e^{-|t-y|}|\phi(t)| d t \leqq \int_{|t-y| \geq \delta} e^{-|t|+\left|\nu_{0}\right|+\delta}|\phi(t)| d t
\end{gathered}
$$

when $\left|y-y_{0}\right| \leqq \delta$. If we now choose $x$ so small that $K \sin x<\epsilon / 2$, the proof is complete.

3. Uniqueness. We inquire next about the character of a function which is harmonic in a strip and zero on the boundary.

THEOREM 2. If

$$
\begin{array}{lr}
\text { 1. } u(x, y) \in H & 0<x<\pi ; \\
\text { 2. } u(x, y) \in C & 0 \leqq x \leqq \pi ; \\
\text { 3. } u(0, y)=u(\pi, y)=0 & -\infty<y<\infty,
\end{array}
$$

then

$$
u(x, y)=\sum_{k=1}^{\infty}\left(A_{k} e^{k y}+B_{k} e^{-k y}\right) \sin k x,
$$

where the series converges for all $x$ and $y$ and

$$
A_{k} e^{k y}+B_{k} e^{-k y}=\frac{2}{\pi} \int_{0}^{\pi} u(x, y) \sin k x d x .
$$

Define $u(x, y)$ for $-\pi \leqq x<0$ by the equation $u(-x, y)=-u(x, y)$. By the Schwarz reflection principle $u(x, y) \in H$ in $-\pi \leqq x \leqq \pi$. By hypothesis $3, u(x, y)$ has the period $2 \pi$ as a function of $x$. Consequently for each fixed $y$ it has an expansion in a Fourier sine-series:

$$
\begin{aligned}
u(x, y) & =\sum_{k=1}^{\infty} C_{k}(y) \sin k x & -\pi \leqq x \leqq \pi ; \\
C_{k}(y) & =\frac{2}{\pi} \int_{0}^{\pi} u(x, y) \sin k x d x, & k=1,2, \cdots .
\end{aligned}
$$

Differentiating (6) twice and using hypothesis 1 , we have 


$$
\begin{aligned}
C_{k}^{\prime \prime}(y) & =-\frac{2}{\pi} \int_{0}^{\pi} u_{x x}(x, y) \sin k x d x \\
& =\frac{2 k^{2}}{\pi} \int_{0}^{\pi} u(x, y) \sin k x d x=k^{2} C_{k}(y) .
\end{aligned}
$$

Here we have integrated by parts, using hypothesis 3 . Solving the resulting differential equation, the result is established.

It should be observed that hypothesis 2 is an essential feature of the theorem. It was used in the application of the reflection principle. Note that the function $P_{y}(x, y) \in H$ in $0<x<\pi$ and that $P_{y}(0+, y)$ $=P_{y}(\pi-, 0)=0$ for all $y$. But it has a singularity at the origin and cannot have the expansion (4). Indeed the integrals (5) all diverge if $u=P_{y}$.

CoRollary 2.1. If, in addition,

$$
\text { 4. } \int_{0}^{\pi}|u(x, y)| d x=O\left(e^{a|y|}\right) \quad|y| \rightarrow \infty,
$$

then

$$
u(x, y)=\sum_{k=1}^{[a]}\left[A_{k} e^{k y}+B_{k} e^{-k y}\right] \sin k x .
$$

For, by hypothesis 4 and equation (5)

$$
A_{k} e^{k y}+B_{k} e^{-k y}=O\left(e^{a|y|}\right) \quad|y| \rightarrow \infty .
$$

If $k>[a]$, this is clearly a contradiction unless $A_{k}=B_{k}=0$.

Corollary 2.2. If
4. $u(x, y) \geqq 0$
$0<x<\pi$

then

$$
u(x, y)=\left[A_{1} e^{y}+B_{1} e^{-y}\right] \sin x,
$$

where $A_{1}$ and $B_{1}$ are non-negative constants.

For, the inequality $|\sin k x| \leqq k|\sin x|, k=1,2, \cdots$, gives

$$
\left|A_{k} e^{k y}+B_{k} e^{-k y}\right| \leqq \frac{2 k}{\pi} \int_{0}^{\pi} u(x, y) \sin x d x=k\left[A_{1} e^{y}+B_{1} e^{-y}\right] .
$$

It is now easy to give a set of conditions which will guarantee the unique representation (2). For example, we have 
Theorem 3. If $u(x, y) \in H$ in $0<x<\pi, \in C$ in $0 \leqq x \leqq \pi, u(0, y) e^{-|y|}$ $\in L, u(\pi, y) e^{-|y|} \in L$, and

$$
\int_{0}^{\pi}|u(x, y)| d x=o\left(e^{|y|}\right) \quad|y| \rightarrow \infty,
$$

then equation (2) holds.

For, by Theorem 1 , the difference between $u(x, y)$ and the integrals (2) satisfies all the conditions of Theorem 2. This difference must be identically zero, since the condition (7) implies that all terms of the series (4) are zero.

Corollary 3.1. Equation (2) holds if $u(x, y) \in H$ in $0 \leqq x \leqq \pi$ and

$$
u(x, y)=O\left(e^{a|y|}\right) \quad a<1,|y| \rightarrow \infty
$$

uniformly in $0 \leqq x \leqq \pi$.

4. Positive harmonic functions. A function harmonic and positive for $0<x<\pi$ has a Poisson-Stieltjes representation, as set forth in the following theorem.

TheOREM 4. A necessary and sufficient condition that $u(x, y) \in H$, and $u(x, y) \geqq 0$ for $0<x<\pi$ is that

$$
\begin{gathered}
u(x, y)=\left[A e^{y}+B e^{-y}\right] \sin x+\frac{1}{2 \pi} \int_{-\infty}^{\infty} P(x, t-y) d \alpha(t) \\
+\frac{1}{2 \pi} \int_{-\infty}^{\infty} P(\pi-x, t-y) d \beta(t),
\end{gathered}
$$

where $A \geqq 0, B \geqq 0, \alpha(t)$ and $\beta(t)$ are nondecreasing, and the integral converges for $0<x<\pi,-\infty<y<\infty$.

This result may be proved in a variety of ways. Perhaps the simplest is by use of the map

$$
\zeta=\xi+i \eta=e^{i(x+i y)}=e^{-y}(\cos x+i \sin x),
$$

which carries the strip $0<x<\pi$ into the half-plane $\eta>0$. Let $F(\xi, \eta)$ be defined by the equation

$$
F\left(e^{-y} \cos x, e^{-y} \sin x\right)=u(x, y) .
$$

Then $F(\xi, \eta) \in H$ and $\geqq 0$ for $\eta>0$, and we may appeal to the Herglotz theorem for the half-plane [4]:

$$
F(\xi, \eta)=P \eta+\frac{1}{\pi} \int_{-\infty}^{\infty} \frac{\eta d \mu(\sigma)}{\eta^{2}+(\sigma-\xi)^{2}} \quad \eta>0
$$


where $P$ is a non-negative constant and $\mu(\sigma)$ is nondecreasing. Break the integral into two parts corresponding to the intervals $(0, \infty)$ and $(-\infty, 0)$. Then

$$
\int_{0}^{\infty} \frac{\eta d \mu(\sigma)}{\eta^{2}+(\sigma-\xi)^{2}}=\frac{\eta[\mu(0+)-\mu(0)]}{\eta^{2}+\xi^{2}}+\int_{0+}^{\infty} \frac{\eta d \mu(\sigma)}{\eta^{2}+(\sigma-\xi)^{2}} .
$$

In the last integral set $\sigma=e^{-t}$ and replace the variables $\xi, \eta$ by their values in terms of $x$ and $y$. It becomes

$$
\frac{1}{2} \int_{-\infty}^{\infty} P(x, t-y) d \alpha(t), \quad d \alpha(t)=-e^{t} d \mu\left(e^{-t}\right) .
$$

The integral (8) extended over the interval $(-\infty, 0)$ is treated similarly, this time by the change of variable $\sigma=-e^{-t}$. Thus equation (9) becomes (8) with

$$
B=P, \quad A=\frac{\mu(0+)-\mu(0-)}{\pi}, \quad d \beta(t)=e^{t} d \mu\left(-e^{-t}\right) .
$$

Clearly $\alpha(t)$ and $\beta(t)$ are nondecreasing, and the proof is complete.

\section{BIBLIOGRAPHY}

1. R. P. Boas, Representations for completely convex functions, Amer. J. Math. vol. 81 (1959) pp. 709-714.

2. G. H. Hardy, A theorem concerning harmonic functions, J. London Math. Soc. vol. 1 (1926) pp. 130-131.

3. G. Herglotz, Über Potenzreihen mit positivem reelen Teil im Einheitskreis, Berichte der Sächsischen Gesellschaft der Wissenschaften zu Leipzig, MathematischPhysikalische Klasse vol. 63 (1911) pp. 501-511.

4. L. H. Loomis and D. V. Widder, The Poisson integral representation of functions which are positive and harmonic in a half-plane, Duke Math. J. vol. 9 (1942) pp. 643645.

5. F. Riesz, Sur certains systèmes singuliers d'équations intégrales, Annales Scientifiques de l'Ecole Normale vol. 28 (1911) pp. 33-62.

HARVARD UNIVERSITY 